\title{
Correspondence
}

\section{Coronary artery patterns in complete transposition} Sir,-In the August 1978 issue of your journal Yacoub and Radley-Smith describe the coronary artery patterns in complete transposition. I was disappointed, however, to see that they gave no reference to previous detailed surveys on this topic. For example, the coronary artery patterns in 89 examples of complete transposition (from a large series of more complex "transpositions") were studied by Rowlatt (1962). Sixty specimens were described by Elliott et al (1963), and 149 examples were reported by Shaher and Puddu (1966) and later redescribed by Shaher in his excellent monograph on complete transposition (1973). The first two studies of coronary artery pattern (Rowlatt, 1962; Elliott et al, 1963) were conducted specifically because of the anticipated possibility of correction of complete transposition by "switching" the great arteries, the very reason for the present report by Yacoub and RadleySmith (1978) except that the correction is no longer anticipated. The reports of Shaher and his colleagues (1966; 1973) are particularly significant. They reported nine coronary arterial patterns in complete transposition. Some of these patterns surely would have been of major importance to any surgeon attempting anatomical correction. For example, a single coronary artery was found in 11 specimens, taking origin from any of the coronary sinuses when viewing this subgroup as a whole. In seven specimens both ostia arose from the same sinus. These variations could well have presented problems in corrective surgery, yet all were not encountered in the series reported by Yacoub and Radley-Smith. Would not this important work have been simply and considerably enhanced by making reference to some of the reports I have mentioned above?

R H ANDERSON

Cardiothoracic Institute, London SW3 6HP.

\section{References}

Elliott, L P, Neufeld, H N, Anderson, R C, Adams, P jun, and Edwards, J E (1963). Complete trans- position of the great vessels. I. An anatomic study of sixty cases. Circulation, 27, 1105-1117.

Rowlatt, U F (1962). Coronary artery distribution in complete transposition. Journal of the American Medical Association, 179, 269-278.

Shaher, R M, and Puddu, G C (1966). Coronary arterial anatomy in complete transposition of the great vessels. American Journal of Cardiology, 17, 355-361.

Shaher, R M (1973). Complete Transposition of the Great Arteries, pp 138-152. Academic Press, New York.

Yacoub, M H, and Radley-Smith, R (1978). Anatomy of the coronary arteries in transposition of the great arteries and methods for their transfer in anatomical correction. Thorax, 33, 418-424.

REPLY - We appreciate Dr Anderson's comments and agree that the articles of doctors Shaher, Elliott, Rowlatt, and colleagues are indeed excellent. Our study was based entirely on a surgical series, and the classification of coronary anatomy suggested relates directly to the methods of their transfer that we need. The methods of transfer described in our paper can be adapted to any variant of the main types of coronary anatomy, including those mentioned by Dr Anderson so long as the following principles are adhered to:

(1) The coronary ostia are not unduly rotated, or under tension, in their new position, particularly in cases of single coronary ostium or early bifurcation. This could be achieved by using method No 2 described in the paper with or without the use of free patches of pulmonary arterial wall to extend the new sinuses giving rise to the coronary arteries.

(2) The proximal part of the coronary arteries in their new position should not pass in close relation to the outside of one of the aortic commissures.

MAGDI YACOUB,

ROSEMARY RADLEY-SMITH 